\title{
LES PARCOURS DE TRANSITION D'UNGERSHEIM : ÊTRE ASPIRÉ PAR LE CHAOS DES CRISES OU EMPRUNTER DES TRAJECTOIRES DE RESILIENCES
}

\author{
Auteur : \\ Guy KONINCKX \\ Senior Consultant \\ G.I.R.O.S., Groupe d'intervention et de recherche en organisation des systèmes \\ Guy.koninckx@skynet.be
}

\begin{abstract}
Résumé :
Une lecture systémique des événements auxquels la commune d'Ungersheim a été confrontée donne accès à une compréhension du fonctionnement de cette collectivité en situation de crise. Les éléments des contextes géographiques et historiques situent le cadre dans lequel elle évolue au cours des vingt dernières années. Des stratégies mises en place par Jean-Claude Mensch, maire d'Ungersheim, ont permis d'opérer une succession d'ajustements. Ceux-ci ont contribué à faire échec et mat à l'effondrement au bénéfice d'une mutation. La cohérence entre la vision et les actions initiées ainsi qu'une règle mythique enracinée apparaissent constituer un élément déterminant, même en situation de turbulences. Ungersheim permet de découvrir les voies de résilience d'une collectivité. Cette commune s'inscrit dans le mouvement de la transition initiée par Hopkins et propose des voies non pas pour échapper aux crises mais plutôt pour les affronter.
\end{abstract}

Mots-clés

crise, stratégies de changements, résiliences.

\section{INTRODUCTION}

Ungersheim, une commune d'Alsace, située à $30 \mathrm{~km}$ d'Allemagne et de Suisse, est confrontée aux conséquences de la fermeture des mines domaniales de potasse d'Alsace. Celles-ci occupaient encore un millier de personnes au début du siècle.

Le parcours est réalisé au départ d'une rencontre avec Jean-Claude Munsch, maire d'Ungersheim. Il exerce son $5^{\mathrm{e}}$ mandat, la $26^{\mathrm{e}}$ année de fonction. Cette rencontre est complétée par des échanges avec l'habitant.

\section{Les contextes géographique et historique en bref}

Ungersheim est situé à $8 \mathrm{~km}$ du massif vosgien. Une dérivation de la Thur alimente l'ancien moulin. Il est loin d'être l'unique. De nombreux moulins font partie du paysage. La cité minière « du moulin » en témoigne.

Ce village de 2.000 habitants dépendait pendant de longues années de l'extraction de la potasse, utilisée pour fabriquer du potassium à des fins agricoles et pour produire du chlorure de sodium (sel) pour les routes en hiver. L'industrie employait 13000 personnes dans la région. La dernière mine a fermé en 2003. 


\section{Repères et points clés}

Tableau $1 \mathrm{Au}$ fil du temps

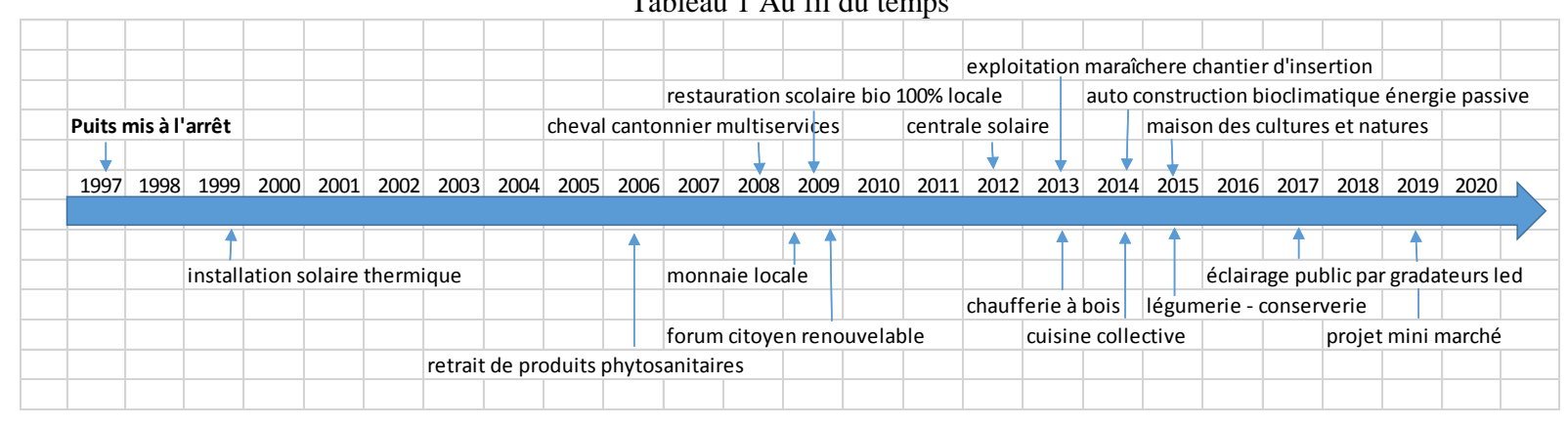

\section{LECTURE DU SYSTÈME UNGERSHEIM}

Une lecture systémique des trajectoires de la commune permet d'aborder son fonctionnement, d'identifier quelques stratégies qui ont permis l'émergence de futurs, de repérer des voies de résilience. C'est dans un contexte de déclin décrit ci avant que s'enracine la vision éclairée de Jean-Claude Mensch. Les éléments clés de celle-ci peuvent se résumer à trois axes essentiels : ramener l'homme à l'humain, s'inscrire dans la post croissance et développer un autre rapport au temps.

Pour donner forme à son intuition, il vise à créer des liens au sein de l'entité, à développer l'autonomie énergétique, à mettre en place une culture axée sur une alimentation saine et travailler avec des personnes relais ainsi qu'un groupe ressource.

Nous constatons la présence de niveaux d'emboîtements pour rendre la vision opérationnelle :

- L'orientation générale au niveau d'Ungersheim, le système. Celle-ci s'inscrit dans le long terme

- Les objectifs pour que l'orientation générale se matérialise s'inscrivent dans le moyen terme

- Les actions pour opérationnaliser les objectifs se déroulent dans le court terme.

Le tableau ci-dessous liste chronologiquement les projets qui ont pris forme. Au fur et à mesure qu'ils sont initiés, les interactions se démultiplient et interagissent au niveau de l'ensemble des actions en cours, une pépinière à projets.

Tableau 2 Les projets et les ministères

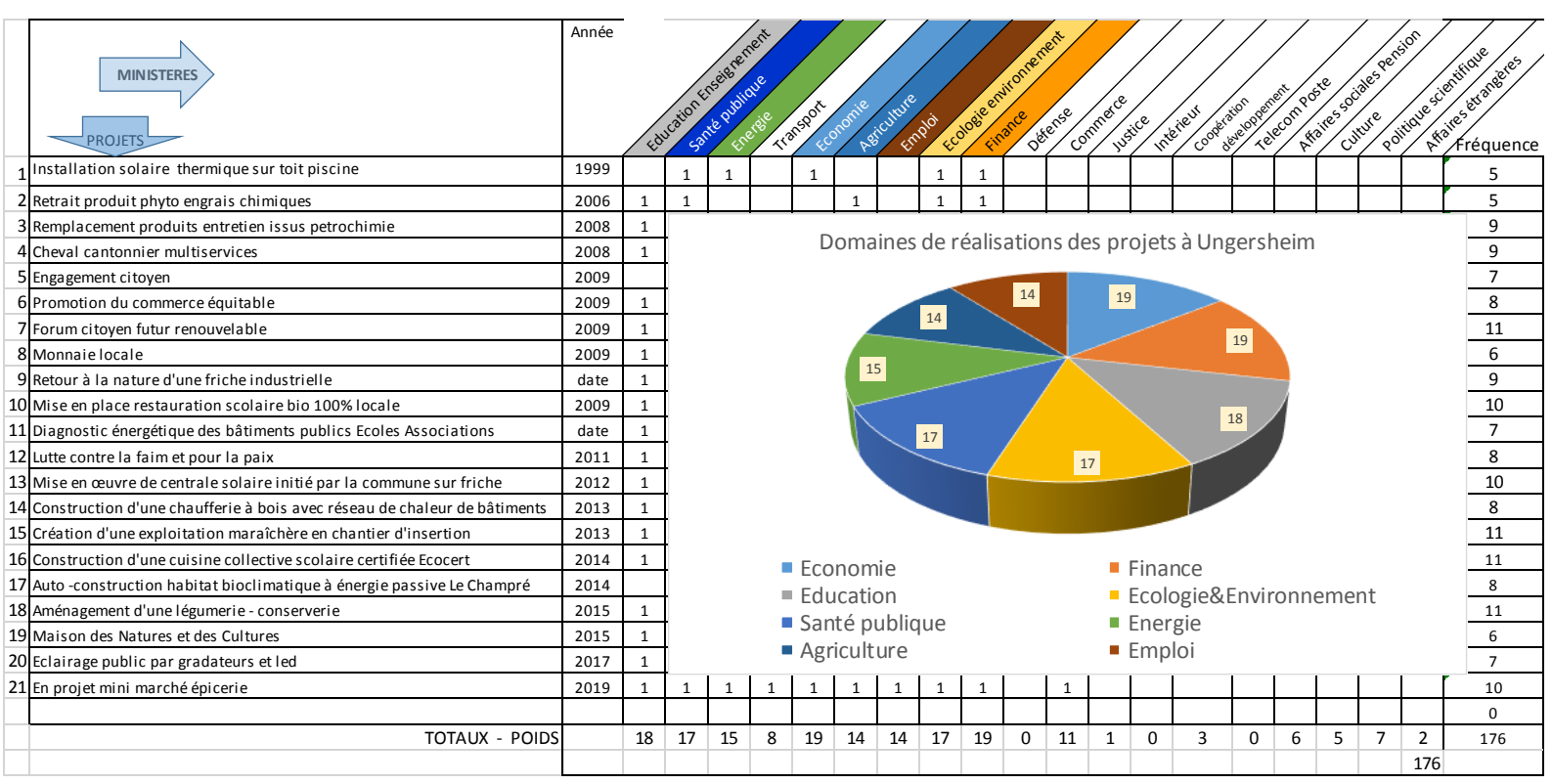


Les différents projets figurent dans la colonne de gauche du tableau ci-dessus. Il liste chronologiquement l'ensemble des 21 projets initiés depuis 1999. En abscisse sont mentionnés les domaines considérés comme des matières ministérielles: l'éducation enseignement, la santé publique, l'énergie, les transports, l'économie, l'agriculture, l'emploi, l'écologie et environnement, la finance, la défense, le commerce, la justice, l'intérieur, la coopération et développement, les télécoms et poste, les affaires sociales, la culture, la politique scientifique et les affaires étrangères.

Les items mentionnés en abscisse et ordonnées ont été croisés. Dans la mesure où un lien existe entre le projet et le domaine d'action de type ministériel nous enregistrons l'existence d'un lien prévisible. Sur cette base de cette liste, nous disposons d'un indicateur établissant un ordre de priorité. Deux groupes émergent: le premier concerne des domaines prioritaires et le second des domaines importants.

- Les priorités sont l'économie, la finance, l'éducation, l'écologie et l'environnement ainsi que la santé publique.

- Importants sans être prioritaires, citons l'énergie, l'agriculture et l'emploi.

- Figurent faiblement ou pas du tout au tableau la justice, l'intérieur, la coopération et développement, les télécoms et la poste, les affaires sociales, la culture, la politique scientifique, la défense et les affaires étrangères.

- Au niveau d'un entre-deux nous trouvons le commerce, le transport, la politique scientifique et les affaires sociales.

Il est à remarquer que tout en étant de nature différente ces projets développent nombre d'interrelations. En laissant la parole aux statistiques $70 \%$ des projets sont en interaction de manière active au niveau de la gestion de la cité. De manière contrastée $15 \%$ restent non abordés au niveau « ministériel ». Il s'agit de la défense, l'intérieur, la poste \& télécommunications. Nous formulons l'hypothèse que le maillage joue un rôle majeur pour pouvoir être considéré comme un système fonctionnel.

Le degré d'amplitude du projet est défini par le nombre de domaines concernés par celui-ci :

Des 21 projets en émergent en particulier 6 :

- Le forum citoyen en 2009.

- La mise en place de la restauration scolaire bio, 100\% locale en 2009.

- La mise en œuvre d'une centrale solaire initiée par la commune sur friche industrielle en 2012.

- La création d'une exploitation maraîchère en chantier d'insertion en 2013.

- La construction d'une cuisine collective scolaire certifiée Ecocert en 2014.

- L'aménagement d'une légumerie - conserverie en 2015.

Nous observons que ces projets, d'une part relèvent de domaines différents et, d'autre part s'étalent dans le temps. Ils s'impriment dans le quotidien d'Ungersheim.

L'hypothèse formulée sur base de ce constat: les projets sont contributeurs du maintien de la mobilisation des membres au fil du temps, étant donné les phases d'incubation et de réalisation proprement dit. C'est comme si les projets assurent un niveau de mobilisation dans un entre-deux.

La ligne du temps (tableau 1) nous autorise à plusieurs observations. En premier lieu des projets sont réalisés, sans discontinuer, sur une période de 15 ans. Ils sont au nombre de 21 actuellement. Et c'est comme si ceux-ci se voient séquencés sur une période de 2 à trois ans. Une fois le projet initié vient la phase de délégation. A partir de 2008 nous constatons comme une multiplication de projets. C'est comme si une courbe d'apprentissage autorisant l'erreur s'installe et constitue un élément facilitateur pour opérer un changement. Nous formulons l'hypothèse que la continuité dans la démarche d'initiation de projets s'alimentant l'un l'autre participe d'une réelle mutation. 
Au fil du temps le projet initié vient se joindre aux existants et complète en quelque sorte le puzzle. Les projets consolident la crédibilité et la pertinence de la vision du maire. C'est comme si les habitants d'Ungersheim, les membres du système, trouvent le fil rouge des actions menées. Un nombre considérable de volontaires participent dans le cadre de chantiers mis en place. Une culture commune s'élabore et prend racine. Nous nous trouvons dans un contexte favorable au développement de relations complémentaires. L'expression est rendue possible dans le cadre des forums et jurys citoyens. Il est permis de parler d'une forme de démocratie participative où les chantiers et projets sont ponctués par des moments de célébrations, des fêtes échelonnées au fil des saisons.

(Au niveau du financement de la centrale solaire, la mairie a initié une démarche public- privé. Relation complémentaire non rigidifiée)

\section{DE LA REGLE MYTHIQUE AUX COMPORTEMENTS OBSERVABLES ET VICE VERSA}

«La démocratie participative est le ciment du bien-être » affirme le maire ; voilà déclinée une règle mythique soutenant l'ensemble. Cette formulation s'apparente en quelque sorte à un mythe familial. Robert Neurburger parle de «ciment qui unifie la famille et confère au groupe une identité qui le différencie du monde extérieur ». L'entité d'Ungersheim en est une illustration.

Certains de ses membres peuvent approuver ce mythe familial, d'autres membres pourront à l'inverse le contester. La règle mythique va constituer le berceau de croyances traduites à leur tour en valeurs. Les comportements, in fine l'élément observable de la règle mythique, en sont l'illustration. Autrement dit un corpus de croyances influe les valeurs sous-tendant les comportements observables.

Dans le contexte d'Ungersheim, Jean-Claude Munsch affirme que la collectivité est source de bien-être, la croissance est limitée, l'autonomie intellectuelle constitue une ressource. Ces croyances sont à considérer comme des attitudes mentales d'acceptations, de convictions intimes, de propositions tenues pour vrai, d'opinions.

Dans cette communauté qui privilégie la force de proposition et le penser par soi-même trois valeurs clés contribuant à «ramener l'homme à l'humain » sont reconnues: la confiance, la solidarité, la fraternité. Les comportements et les pratiques viennent le confirmer.

Certains membres du système peuvent y voir la négation de leur individualité. Des résistances, voire des oppositions, peuvent se manifester et constituer des sources de tensions.

Dans l'entité d'Ungersheim pointons des modes de fonctionnement récurrent:

- Travailler avec des objectifs

- Fonctionner avec une diversité de projets

- Innover régulièrement dans un cycle : innovation - rétroaction - évaluation

- Organiser des chantiers citoyens basés sur le volontariat

- Ponctuer l'année de moments rassembleurs et faire la fête ensemble

- Fonctionner avec des conseils et jury (participatifs, enfants, sages, citoyens)

- Ne pas rester focalisé sur une résistance

- Prendre le temps dans ce qui est entrepris

- Remettre au premier plan des questions relatives aux objectifs, valeurs, limites de la croissance

La récurrence de ces comportements permet de les considérer comme l'expression de règles de fonctionnement du système Ungersheim. 


\section{DES PARCOURS DE TRANSITION IDENTIFIES A UNGERSHEIM, LES VOIES DE LA RESILIENCE}

La période de fermeture des puits de potasse a été déstabilisante pour la population D'Ungersheim. Une période de reconstruction et de redéploiement de sens a pris le relais.

Faire face, rebondir, amorcer d'autres voies s'apparentent à la résilience, le contraire de la rigidité. Relevons quelques éléments clés des parcours :

- Déployer une stratégie basée sur des actions à court, moyen et long terme cohérentes avec la vision

- La succession d'aménagements s'appuie sur une vision et conduit à une mutation

- Des résistances, freins et croyances font partie du parcours de transition

- Redonner régulièrement une vue sur les différentes actions initiées et en cours

- Retracer au fil du temps des liens permet de retourner au sens

- La pratique des rétroactions permet de réaliser des ajustements utiles pour enraciner la vision

- Assurer une continuité en travaillant avec des personnes relais et un groupe ressource (inspiration)

- Réunir les gens et les inviter à parler autour de thématiques concernant l'avenir de la communauté permet de les impliquer.

- L'école comme lieu d'apprentissage, c'est miser sur l'avenir

- Implication

- Alimentation

- Energie

- Ecologie

Ungersheim fait preuve de résilience collective. Elle se définit comme la capacité d'une collectivité, confrontée à des événements fragilisants et déstabilisants, voire catastrophiques, de mobiliser ses ressources en vue de se redéployer dans des activités en lien avec sa finalité.

Une collectivité n'est pas résiliente en soi ; ce sont les membres qui disposent de cette capacité à mobiliser leurs aptitudes face à une situation déstabilisante. Pour K. Weick ce sont les éléments qui contribuent à l'émergence d'une nouvelle fiabilité, définie comme le reflet de la capacité à s'organiser et à se réorganiser pour faire face à des situations imprévues et dangereuses. Il distingue la construction de sens au niveau individuel de l'élaboration collective du sens. Le lien contribue à tisser l'identité collective. Des relations saines et écologiques constituent le socle pour que le processus de résilience non seulement s'enclenche mais se poursuive.

La résilience ne correspond pas à un état, elle est en évolution constante. A Ungersheim, la perspective temps s'inscrit résolument dans le présent et le futur.

Le fonctionnement de l'entité permet d'identifier des stratégies de rebond et privilégie la cohérence des actions en reliant les parties dans un ensemble plus large.

\section{CONCLUSIONS}

Que retenir de la rencontre de Jean-Claude Mensch - maire d'Ungersheim ?

1. Constitue une pratique de démocratie participative inspirée.

2. Témoigne de la puissance d'une vision inspirante pour les objectifs et les actions.

3. Intègre réflexion et action.

4. Connecte la praxis et la doxa.

5. Articule des niveaux de décision.

6. Accepte de marcher à tâtons dans la perspective de post croissance.

7. Prend le risque d'évoluer dans un entre-deux. 
8. Pose la question des enjeux de la finalité.

9. Témoigne des ressources de la résilience individuelle et collective.

\section{RÉFÉRENCES}

IONESCU, S. (2011). Traîté de résilience assistée. Presses universitaires de France.

KONINCKX, G. \& TENEAU, G. (2010). Résilience organisationnelle - Rebondir face aux turbulences. De Boeck, Bruxelles.

PIECQ, A. (2011). De la pensée systémiue à la pratique de l'organisation. L'Harmattan, Paris.

WEICK, K. (1995) Sensemaking in organizations. Thousand Oaks, CA, Sage publications, Fondations for organizational science.

WEICK, K. \& STCLIFFE, K. M. (2007) Managing the Unexpected: Resilient Performance in an Age of Uncertainty ( $2^{\mathrm{e}}$ éd.) San Francisco: Jossey-Bass.

Collectif (2016). Résiliences, Ressemblances dans la diversité. Odile Jacob, Paris. 